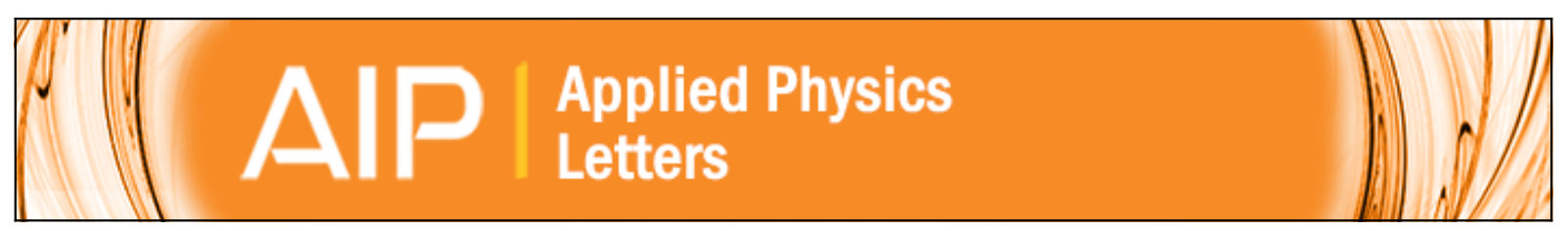

\title{
Enhancement of p-type mobility in tin monoxide by native defects
}

D. B. Granato, J. A. Caraveo-Frescas, H. N. Alshareef, and U. Schwingenschlögl

Citation: Applied Physics Letters 102, 212105 (2013); doi: 10.1063/1.4808382

View online: http://dx.doi.org/10.1063/1.4808382

View Table of Contents: http://scitation.aip.org/content/aip/journal/apl/102/21?ver=pdfcov

Published by the AIP Publishing 


\title{
Enhancement of p-type mobility in tin monoxide by native defects
}

\author{
D. B. Granato, J. A. Caraveo-Frescas, H. N. Alshareef, and U. Schwingenschlögla) \\ KAUST, Physical Science and Engineering Division, Thuwal 23955-6900, Kingdom of Saudi Arabia
}

(Received 7 March 2013; accepted 17 May 2013; published online 31 May 2013)

\begin{abstract}
Transparent p-type materials with good mobility are needed to build completely transparent $\mathrm{p}-\mathrm{n}$ junctions. Tin monoxide $(\mathrm{SnO})$ is a promising candidate. A recent study indicates great enhancement of the hole mobility of $\mathrm{SnO}$ grown in Sn-rich environment [E. Fortunato et al., Appl. Phys. Lett. 97, 052105 (2010)]. Because such an environment makes the formation of defects very likely, we study defect effects on the electronic structure to explain the increased mobility. We find that $\mathrm{Sn}$ interstitials and $\mathrm{O}$ vacancies modify the valence band, inducing higher contributions of the delocalized Sn $5 p$ orbitals as compared to the localized $\mathrm{O} 2 p$ orbitals, thus increasing the mobility. This mechanism of valence band modification paves the way to a systematic improvement of transparent p-type semiconductors. @ 2013 AIP Publishing LLC. [http://dx.doi.org/10.1063/1.4808382]
\end{abstract}

Transparent oxide semiconductors are materials that combine controllable electrical conductivity with optical transparency. ${ }^{1}$ Though broadly used in a range of devices, from solar cells to organic light emitting diodes, the application of transparent semiconductors is limited because of a lack of p-type materials with good properties, ${ }^{2,3}$ such as high hole mobility and controllable hole density. Good p-type transparent oxide semiconductors would allow the fabrication of transparent p-n junctions, enabling new applications of invisible circuits. ${ }^{4-6}$ However, nowadays, the materials still suffer from a low conductivity as compared to their n-type counterparts. ${ }^{7,8}$ Therefore, it is crucial to find ways to improve the hole transport.

The fact that hole transport in oxides is much worse than electron transport is determined by the states forming the edges of the valence and conduction bands. Typically, the edge of the valence band (VB) is dominated by the $\mathrm{O} 2 p$ states, while the edge of the conduction band $(\mathrm{CB})$ is governed by the metal states, as exemplified in the left-hand side of Figure 1. This configuration renders a high mobility for the electrons in the $\mathrm{CB}$, whereas the mobility of the holes in the VB is poor as they are trapped in localized O $2 p$ orbitals. With this picture in mind, a chemical design of p-type oxides was proposed..$^{9,10}$ The main idea is to choose a metal possessing a closed or pseudo closed shell with an energy near to that of the $\mathrm{O} 2 p$ states, which increases the contribution of the delocalized metal states to the VB edge and thus improves the hole mobility. This mechanism is greatly exemplified by tin monoxide $(\mathrm{SnO})$, which shows a high contribution of delocalized Sn $5 s$ states to the VB and of likewise delocalized Sn $5 p$ states to the CB (right-hand side of Figure 1), making the material a promising candidate for transparent applications. ${ }^{7,11,12}$

In spite of great improvements in recent years, an even better hole mobility is required for more complex applications. In this direction, recent reports have demonstrated that $\mathrm{SnO}$ films deposited in a very Sn-rich environment exhibit a high hole mobility. ${ }^{5,12}$ It has been suggested that the enhancement of the hole mobility is due to the presence of

a)udo.schwingenschlogl@kaust.edu.sa. Tel.: +966(0)544700080 metallic $\beta$-Sn. However, because such a Sn-rich environment is also likely to cause a lot of defects, we will take a deeper look at the defects' behavior. A theoretical study on defects in $\mathrm{SnO}$ has shown that $\mathrm{Sn}$ vacancies in the -2 charged state are responsible for the p-type behavior and has analysed the charge distribution around the defects, ${ }^{13}$ but there is no study reported on the impact of defects on the mobility. We attempt to explain and rationalize the enhancement of the hole mobility of $\mathrm{SnO}$ grown in Sn-rich environment by first principles calculations, analysing the effects of native defects on the orbital composition of the electronic states.

We calculate electronic structures in the framework of density functional theory using the projector-augmented wave method ${ }^{14,15}$ as implemented in the Vienna Ab-initio Simulation Package. ${ }^{16,17}$ The electron-electron interaction is treated in the generalized gradient approximation as parametrized by Perdew et al. ${ }^{18}$ The Sn $5 s, 5 p$, and $4 d$ as well as the $\mathrm{O} 2 s$ and $2 p$ orbitals are considered as valence states, whereas all other orbitals are treated as core states. The energy cutoff is set to $600 \mathrm{eV}$ and the Brillouin zone sampled using the Monkhorst-Pack method ${ }^{19}$ with a $\Gamma$-centered $3 \times 3 \times 3 \mathrm{k}$-mesh during the self-consistency cycle. The atomic forces and total energy are converged to less than $0.02 \mathrm{eV} \AA^{-1}$ and $10^{-5} \mathrm{eV}$, respectively. All the numerical parameters are selected after a careful convergence analysis.

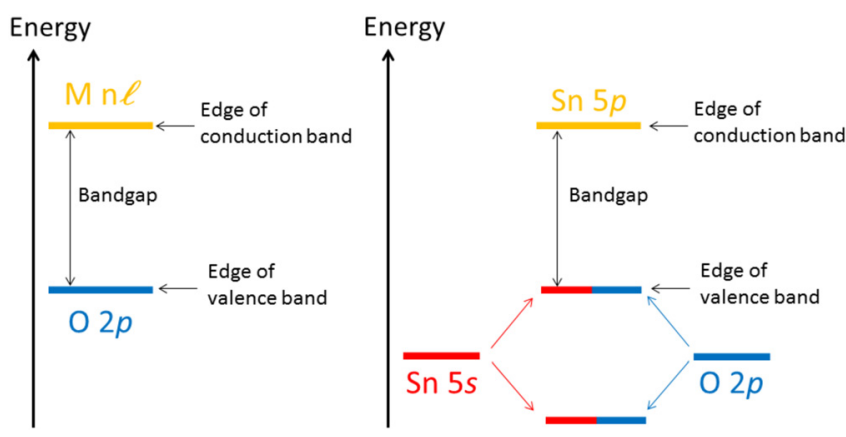

FIG. 1. Schematic diagram of the energy levels for a typical metal oxide (left) and for $\mathrm{SnO}$ (right). For the typical metal oxide, the VB is dominated by the $\mathrm{O} 2 p$ orbitals and the $\mathrm{CB}$ by the cation states (e.g., $\mathrm{Sn} 5 s$ for $\mathrm{SnO}_{2}$ ). For $\mathrm{SnO}$, the energy proximity of the $\mathrm{Sn} 5 s$ and $\mathrm{O} 2 p$ states results in equal contributions to the VB edge. For a more complete picture of the level energetics, the reader is referred to Refs. 24 and 25 . 
To calculate the electronic density of states (DOS), the $\mathrm{k}$-mesh is refined to $7 \times 7 \times 7$ and the tetrahedron integration method is used. Partial DOSs are evaluated by projecting the wave functions onto spherical harmonics around the Sn and $\mathrm{O}$ atoms within a radius of 1.59 and $1.34 \AA$, respectively. These values are selected by means of a Bader charge analysis using the code of Henkelman and coworkers ${ }^{20,21}$ in order to minimize both the overlap between the spheres and the charge in the interstitial regions not covered by the spheres.

$\mathrm{SnO}$ has a litharge tetragonal structure (space group no. 129) with two formula units per unit cell. Our optimized lattice parameters of the pristine $\mathrm{SnO}$ unit cell are $a=3.87 \AA$ and $c=5.06 \AA$, which compare well with the experimental values $^{22}$ and with previous theoretical work on $\mathrm{SnO}$ using the generalized gradient approximation. ${ }^{13,23}$ Defects are simulated using a $3 \times 3 \times 2$ supercell with 72 atoms modified by one of the following: a vacancy of tin $\left(\mathrm{V}_{\mathrm{Sn}}\right)$, an interstitial of oxygen $\left(\mathrm{O}_{\mathrm{i}}\right)$, a vacancy of oxygen $\left(\mathrm{V}_{\mathrm{O}}\right)$, or an interstitial of tin $\left(\mathrm{Sn}_{\mathrm{i}}\right)$. For the interstitials, there are two possible sites: The first is a tetrahedral site surrounded by four $\mathrm{Sn}$ atoms and the second is (almost) the center of a Sn octahedron (offset along the $\mathrm{z}$-axis away from the $\mathrm{O}$ plane), as shown in Figure 2. The present work only considers the octahedral site, which was shown to be the most stable interstitial site. ${ }^{13}$ For the defective supercells, the lattice parameters are kept fixed at the values calculated for the pristine cell and only the atomic positions are optimized.

The orbital contributions to the electronic structure of pristine $\mathrm{SnO}$ are visualized by the partial DOSs in Figure 3, showing a hybridization of the $\mathrm{O} 2 p$, Sn $5 s$, and Sn $5 p$ states in the VB. The $\mathrm{O} 2 p$ and $\mathrm{Sn} 5 s$ states form bonding and antibonding orbitals that are completely filled. Normally, this would not be energetically favorable, but a symmetry breaking in the z-direction allows for a further hybridization with the Sn $5 p$ orbitals (seen in the partial DOS), which lowers the total energy. This mechanism gives rise to the SnO litharge structure as pointed out by Walsh and Watson. ${ }^{24,25}$

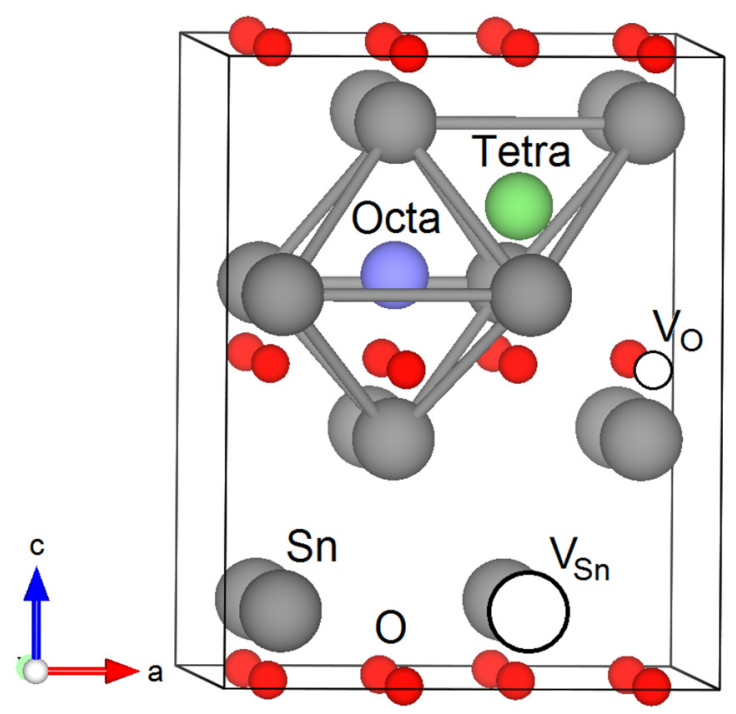

FIG. 2. SnO native defects. Sn atoms are represented by large grey spheres and $\mathrm{O}$ atoms by small red spheres. The octahedral site is shown in blue and the tetrahedral site in green. Only the octahedral site is considered in this work.
Now, to understand the charge transport, we have to examine the band edges because there the transport takes place. The edge of the VB is located at $0 \mathrm{eV}$ (Fermi energy) and is composed of $\mathrm{Sn} 5 s$ and O $2 p$ states (approximately 40:60 mixing). The $\mathrm{CB}$ edge is located near $0.5 \mathrm{eV}$ and is dominated by $\mathrm{Sn} 5 p$ states. These results are consistent with the principal picture discussed previously and with previous theoretical works. ${ }^{13,24,25}$ Because the metal states are more spatially spread than the oxygen states, the metallic contribution to the VB renders the good hole mobility of $\mathrm{SnO} .^{9,11,12}$ Therefore, an increase in the metallic contributions to the VB edge is expected to further enhance the hole mobility.

Figure 4 presents the calculated electronic structures of $\mathrm{SnO}$ with $\mathrm{V}_{\mathrm{Sn}}, \mathrm{O}_{\mathrm{i}}, \mathrm{V}_{\mathrm{O}}$, and $\mathrm{Sn}_{\mathrm{i}}$ defects, focusing on the region from -2 to $2 \mathrm{eV}$. When introducing a $\mathrm{V}_{\mathrm{Sn}}$ in $\mathrm{SnO}$, we observe a lowering of the Fermi level, indicating an acceptor behavior for this defect. This observation is consistent with the results of the formation energy study in Ref. 13. Concerning the VB, however, there is no substantial change as compared to the pristine case, with the edge still being composed of a 40:60 mixture of Sn $5 s$ and O $2 p$ orbitals. Thus, apart from $\mathrm{V}_{\mathrm{Sn}}$ being an acceptor, this defect does not affect much the electronic behavior of the VB edge. Likewise, for the $\mathrm{O}_{\mathrm{i}}$ defect there is no noticeable change in the VB edge. The $2 p$ orbitals of the interstitial oxygen contribute mostly to the center of the $\mathrm{VB}$, from -4 up to $-0.5 \mathrm{eV}$, approximately. Thus, the $\mathrm{O}_{\mathrm{i}}$ does not affect the edge of the VB and is not expected to change the mobility of the holes.

On the other hand, the $\mathrm{V}_{\mathrm{O}}$ defect shows a strong modification of the VB edge from -0.25 to $0 \mathrm{eV}$. While the contribution of the Sn $5 s$ states to the VB decreases a little, the contribution of the $\mathrm{Sn} 5 p$ states increases considerably. Overall, this enhances the contribution of the $\mathrm{Sn}$ states (Sn $5 s+\mathrm{Sn} 5 p$ ) to the VB edge, which now is about twice the contribution of the $\mathrm{O} 2 p$ states. As discussed previously, this is expected to increase the mobility of the holes because of the more dispersive character of the metallic bands. ${ }^{7,9,11,12}$

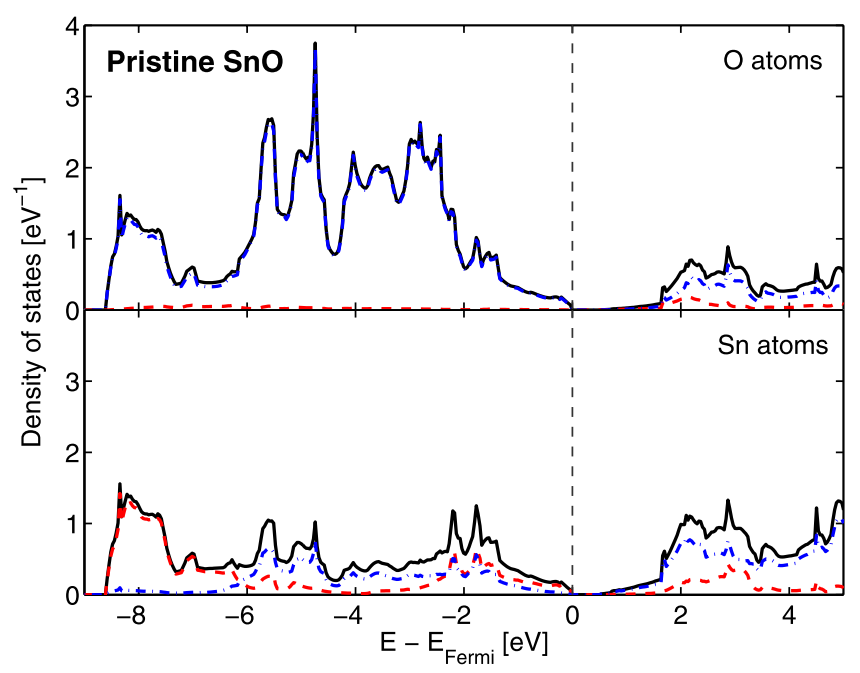

FIG. 3. Partial DOSs of pristine SnO. The summed valence states $(\mathrm{O} 2 s+2 p$ and $\mathrm{Sn} 5 s+5 p+4 d$ ), the summed $s$ states (O $2 s$ and $\mathrm{Sn} 5 s)$, and the summed $p$ states (O $2 p$ and $\mathrm{Sn} 5 p$ ) of all atoms of the same type are represented by a black solid, a red dashed, and a blue dashed-dotted line, respectively. The Fermi energy is set to the top of the valence band. 

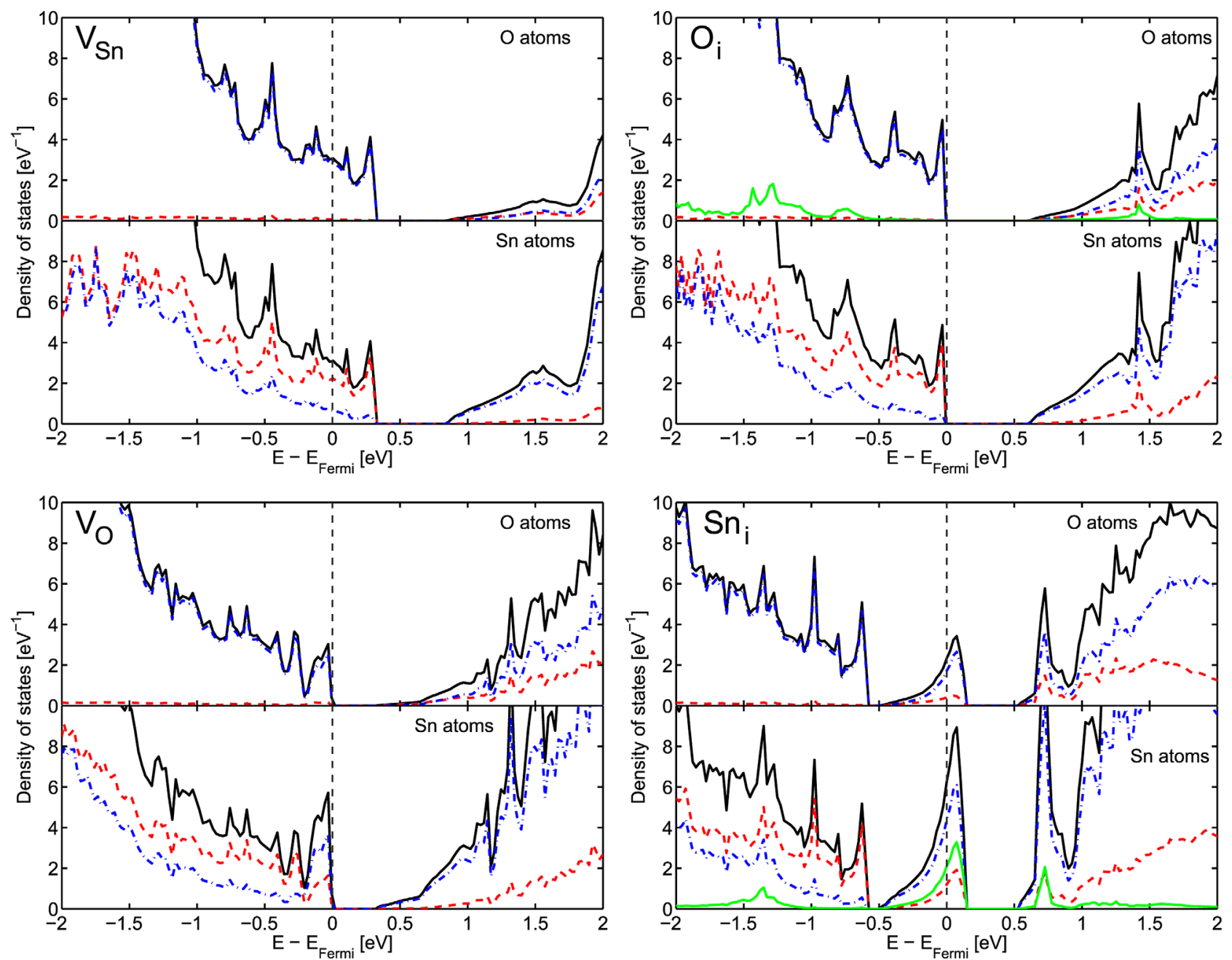

FIG. 4. Partial DOSs for the defective SnO supercells. The summed valence states $(\mathrm{O} 2 s+2 p$ and $\mathrm{Sn} 5 s+5 p+4 d)$, the summed $s$ states $(\mathrm{O} 2 s$ and $\mathrm{Sn} 5 s)$, and the summed $p$ states ( $\mathrm{O} 2 p$ and $\mathrm{Sn} 5 p$ ) of all atoms of the same type are represented by a black solid, a red dashed, and a blue dashed-dotted line, respectively. A green solid line represents the $p$ states of the interstitial atom, when there is one. The energy is measured in relation to the Fermi energy represented by a vertical dashed line.

A similar feature is also observed in the DOS of the $\mathrm{Sn}_{\mathrm{i}}$ defect. The state created by the $\mathrm{Sn}_{\mathrm{i}}$ near the VB edge shows equal contributions from the $5 p$ orbitals of the interstitial $\mathrm{Sn}$ and from the $\mathrm{O} 2 p$ orbitals. However, there is also a substantial contribution from the $5 p$ orbitals of the lattice Sn atoms (not shown) resulting in a metal dominated region near the VB edge, thus enhancing the overall hole mobility. Similar or even better modifications of the VB edge could, in principle, also be achieved by other dopants.

We note that the $\mathrm{O}_{\mathrm{i}}$ and $\mathrm{V}_{\mathrm{Sn}}$ defects, which do not affect much the electronic structure, are more likely to appear in O-rich environment. On the other hand, $\mathrm{V}_{\mathrm{O}}$ and $\mathrm{Sn}_{\mathrm{i}}$ defects, which have a great impact on the hole mobility by modifying the VB, are more likely to appear in Sn-rich environment, thus explaining the increase in hole mobility of $\mathrm{SnO}$ grown under such conditions, as observed experimentally. ${ }^{12,26}$ A caveat to this is that the presence of defects will also lead to a scattering of the charge carriers such that a high concentration of defects might counteract a high mobility. Therefore, it is important to find a balance between the scattering and the mobility enhancement by electronic modification of the VB edge to obtain an optimal hole mobility.
Using density functional theory, we have studied the effects of native defects on the electronic structure of $\mathrm{SnO}$, a transparent p-type semiconductor. In particular, we have focused on a qualitative explanation of the mobility increase found in $\mathrm{SnO}$ grown in $\mathrm{Sn}$-rich environment by studying the metallic contributions to the VB edge. We find that $\mathrm{O}_{\mathrm{i}}$ and $\mathrm{V}_{\mathrm{Sn}}$ defects, that typically are generated under O-rich growth conditions, do not modify the VB edge and thus are not expected to contribute to the enhancement of the hole mobility. Conversely, the $\mathrm{V}_{\mathrm{O}}$ and $\mathrm{Sn}_{\mathrm{i}}$ defects dominating under Sn-rich growth conditions enhance the metallic character of the VB, mainly due to the Sn $5 p$ states, and thus the overall hole mobility. Therefore, the presence of either $\mathrm{V}_{\mathrm{O}}$ or $\mathrm{Sn}_{\mathrm{i}}$ defects can explain the mobility increase for $\mathrm{SnO}$ grown in Sn-rich environment. Moreover, our results indicate that pursuing dopants that further modify the VB edge with delocalized states can be a way to achieve even higher p-type mobility.

${ }^{1}$ S. Sheng, G. Fang, C. Li, S. Xu, and X. Zhao, Phys. Status Solidi A 203, 1891 (2006).

${ }^{2}$ D. S. Ginley and C. Bright, MRS Bull. 25, 15 (2000).

${ }^{3}$ P. D. C. King and T. D. Veal, J. Phys.: Condens. Matter 23, 334214 (2011).

${ }^{4} \mathrm{G}$. Thomas, Nature 389, 907 (1997). 
${ }^{5}$ E. Fortunato, P. Barquinha, and R. Martins, Adv. Mater. 24, 2945 (2012). ${ }^{6}$ H. Hosono, Y. Ogo, H. Yanagi, and T. Kamiya, Electrochem. Solid State Lett. 14, H13 (2011).

${ }^{7}$ Y. Ogo, H. Hiramatsu, K. Nomura, H. Yanagi, T. Kamiya, M. Hirano, and H. Hosono, Appl. Phys. Lett. 93, 032113 (2008).

${ }^{8}$ E. Fortunato and R. Martins, Phys. Status Solidi RRL 5, 336 (2011).

${ }^{9}$ H. Kawazoe, M. Yasukawa, H. Hyodo, M. Kurita, H. Yanagi, and H. Hosono, Nature 389, 939 (1997).

${ }^{10}$ H. Yanagi, H. Kawazoe, A. Kudo, M. Yasukawa, and H. Osono, J. Electroceram. 4, 407 (2000).

${ }^{11}$ Y. Ogo, H. Hiramatsu, K. Nomura, H. Yanagi, T. Kamiya, M. Kimura, M. Hirano, and H. Hosono, Phys. Status Solidi A 206, 2187 (2009).

${ }^{12}$ E. Fortunato, R. Barros, P. Barquinha, V. Figueiredo, S.-H. K. Park, C.-S. Hwang, and R. Martins, Appl. Phys. Lett. 97, 052105 (2010).

${ }^{13}$ A. Togo, F. Oba, I. Tanaka, and K. Tatsumi, Phys. Rev. B 74, 195128 (2006).

${ }^{14}$ P. E. Blöchl, Phys. Rev. B 50, 17953 (1994).

${ }^{15}$ G. Kresse and D. Joubert, Phys. Rev. B 59, 1758 (1999).
${ }^{16}$ G. Kresse and J. Furthmüller, Comput. Mater. Sci. 6, 15 (1996).

${ }^{17}$ G. Kresse and J. Furthmüller, Phys. Rev. B 54, 11169 (1996).

${ }^{18}$ J. P. Perdew, K. Burke, and M. Ernzerhof, Phys. Rev. Lett. 77, 3865 (1996).

${ }^{19}$ H. J. Monkhorst and J. D. Pack, Phys. Rev. B 13, 5188 (1976).

${ }^{20}$ E. Sanville and S. Kenny, J. Comput. Chem. 28, 899 (2007).

${ }^{21}$ W. Tang, E. Sanville, and G. Henkelman, J. Phys.: Condens. Matter 21, 084204 (2009).

${ }^{22}$ F. Izumi, J. Solid State Chem. 38, 381 (1981).

${ }^{23}$ J. P. Allen, D. O. Scanlon, S. C. Parker, and G. W. Watson, J. Phys. Chem. C 115, 19916 (2011).

${ }^{24}$ A. Walsh and G. W. Watson, Phys. Rev. B 70, 235114 (2004).

${ }^{25}$ A. Walsh and G. W. Watson, J. Phys. Chem. B 109, 18868 (2005).

${ }^{26}$ J. A. Caraveo-Frescas, P. K. Nayak, H. A. Al-Jawhari, D. B. Granato, U. Schwingenschlögl, and H. N. Alshareef, "Record Mobility in Transparent p-Type Tin Monoxide Films and Devices by Phase Engineering," ACS Nano (to be published). 